\title{
Sinus venosus atrial septal defect in a 31- year-old female patient: a case for surgical repair
}

\author{
M.A. Gatzoulis and G. Giannakoulas
}

ABSTRACT: A 31-yr-old female patient previously diagnosed with idiopathic pulmonary arterial hypertension (PAH) was referred to our centre for further evaluation. Cardiac magnetic resonance imaging (MRI) and echocardiography revealed a superior sinus venosus atrial septal defect (ASD) with partial anomalous pulmonary venous drainage. Following re-diagnosis, surgical repair was considered. Despite a disproportionately high mean pulmonary artery pressure $(\bar{P} p a)$ of $47 \mathrm{mmHg}$ relative to the patient's age and defect, the decision to operate was based on the absence of oxygen desaturation (either at rest or during exercise), cyanosis or abnormally elevated haemoglobin. Other operability criteria included normal sinus rhythm at rest, vasoreactivity and a pulmonary to systemic blood flow ratio of 1.9 at rest.

Surgical repair and continued advanced therapy with bosentan $125 \mathrm{mg}$ b.i.d. and aspirin $75 \mathrm{mg}$ o.d. proved successful, with post-operative improvements in exercise capacity and dyspnoea, and a reduction in $\bar{P}$ pa to $25 \mathrm{mmHg}$. Keen to start a family, the risks of pregnancy were discussed. This case illustrates the importance of secondary PAH in sinus venosus ASD and the need to exclude a sinus venosus ASD in unexplained right ventricular dilatation. Access to expertise in congenital heart disease and use of cardiac MRI can improve diagnosis and, in turn, treatment decisions.

KEYWORDS: Atrial septal defect, congenital heart disease, pulmonary arterial hypertension

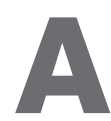
31-yr-old female patient with a long history of idiopathic pulmonary arterial hypertension (IPAH) was referred to the Royal Brompton Hospital, London, UK in 2007 for further evaluation. 8 yrs earlier, the patient had presented at another institution with atypical chest pain and shortness of breath and was subsequently diagnosed with IPAH. Right heart cardiac catheterisation (RHC) performed at that time showed the patient had unexpectedly high oxygen saturation in the pulmonary artery (84\%), but not in the right atrium, which did not change with adenosine challenge (83\%). Pulmonary artery pressure $(\mathrm{Ppa})$ was high, with a mean of $42 \mathrm{mmHg}$, but wedge pressure was normal (mean $5 \mathrm{mmHg}$ ), consistent with a diagnosis of pulmonary arterial hypertension (PAH). Pulmonary vascular resistance (PVR) was also high (4.09 Wood units). Both Ppa and PVR decreased on adenosine challenge, indicating a reactive pulmonary vascular bed. The patient was initially treated with the calcium antagonist diltiazem but failed to respond, and was then switched to oral bosentan. Treatment with bosentan produced excellent symptomatic improvement and was maintained thereafter.

Subsequent to the first diagnosis, the patient was referred to the Royal Brompton Hospital where she underwent cardiac magnetic resonance imaging (MRI), during which a superior sinus venosus atrial septal defect (ASD) with partial anomalous pulmonary venous drainage (PAPVD) was detected (table 1; fig. 1). At the time of the secondary diagnosis in 2007, the patient was in World Health Organization (WHO) functional class II and taking bosentan $125 \mathrm{mg}$ b.i.d. and warfarin.

A series of tests were then carried out to determine the patient's suitability for cardiac surgery to close the septal defect. On examination, the patient was pink $(100 \%$ oxygen saturation), had normal blood pressure (105/65 $\mathrm{mmHg}$ ) and a good volume pulse. The patient

\section{AFFILIATIONS}

Adult Congenital Heart Centre and Centre for Pulmonary Hypertension Royal Brompton Hospital and the National Heart and Lung Institute, Imperial College, London, UK.

\section{CORRESPONDENCE}

M.A. Gatzoulis

Royal Brompton Hospital

Sydney St

London

SW3 6NP

UK

E-mail: m.gatzoulis@rbht.nhs.uk

Received:

Aug 232010

Accepted after revision:

Sept 132010

PROVENANCE

Publication of this peer-reviewed article was supported by Actelion Pharmaceuticals Ltd, Switzerland (unrestricted grant, European Respiratory Review issue 118). 


\section{TABLE 1 Cardiac magnetic resonance imaging scan}

$12 \mathrm{~mm}$ sinus venosus defect with anomalous right upper and middle pulmonary vein drainage

Moderately enlarged right-sided chambers with mild right ventricular hypertrophy

Flattened interventricular septum

Left ventricular chambers of normal size and function

Enlarged pulmonary trunk: $34 \mathrm{~mm}$ (ascending aorta $21 \mathrm{~mm}$ )

Right ventricular end diastolic volume $192 \mathrm{~mL}$

Right ventricular systolic volume $80 \mathrm{~mL}$

Left ventricular diastolic volume $77 \mathrm{~mL}$

Left ventricular systolic volume $29 \mathrm{~mL}$

Cardiac output $3.3 \mathrm{~L} \cdot \mathrm{min}^{-1}$

Pulmonary to systemic blood flow ratio 1.9

also had a prominent right ventricular lift, with a wide and fixed splitting S2 with a loud pulmonary component to it, and a pulmonary ejection systolic murmur of $1-2 / 6$ at the upperleft sternal edge. There was no evidence of decompensation or peripheral oedema. Jugular venous pressure was not particularly elevated and, as a consequence, the chest radiograph was clear. Other relevant information included a haemoglobin concentration of $12.3 \mathrm{~g} \cdot \mathrm{dL}^{-1}$ with no evidence of iron deficiency and anaemia, and an elevated brain natriuretic peptide concentration of $45 \mathrm{pg} \cdot \mathrm{mL}^{-1}$. The patient's ECG at rest showed normal sinus rhythm with normal P-wave axis, QRS-wave axis and narrow QRS complex. This suggested a fairly benign ECG on presentation and, moreover, no evidence of underlying sinus node disease.

Chest radiograph indicated some cardiac abnormalities (fig. 2). There was some enlargement of the right heart, a normal atrial arrangement, greatly enlarged pulmonary arteries (including the presence of a large aneurysm in the central pulmonary artery) and mildly increased pulmonary vascular markings, but no evidence of cardiomegaly as defined by a 50\% cardiothoracic ratio (a prognostic marker for poor survival). Transthoracic echocardiography confirmed the diagnosis of sinus venosus ASD in this patient (fig. 3). The defect was $\sim 20 \mathrm{~mm}$ in size (larger than that shown on MRI), indicating that flow was not too severely restricted, with a left-to-right shunt at rest and an anomalous right superior and middle pulmonary vein into the superior vena cava (SVC), which is not uncommon in such cases. There was moderate right heart enlargement but with preserved right ventricular function, an estimated right ventricular systolic pressure of $57 \mathrm{mmHg}$, and acceleration time of the pulmonary flow of $81 \mathrm{~m} \cdot \mathrm{s}^{-1}$, indicative of elevated PVR. Both the main and branch pulmonary arteries were enlarged. Left ventricular systolic function was maintained, but with reversed septal motion. The aortic arch was normal.

RHC carried out at this time showed high oxygen saturation in the SVC, which decreased on adenosine challenge (table 2). Conversely, the high oxygen saturation in the pulmonary artery increased on vasoreactivity challenge, while PVR decreased slightly. With respect to exercise capacity, the patient achieved a distance of $446 \mathrm{~m}$ in the 6-min walk test (6MWT), during which oxygen saturation decreased only slightly, from $99 \%$ to $97 \%$, heart rate increased from 91 to 101 beats $\cdot \mathrm{min}^{-1}$ and dyspnoea

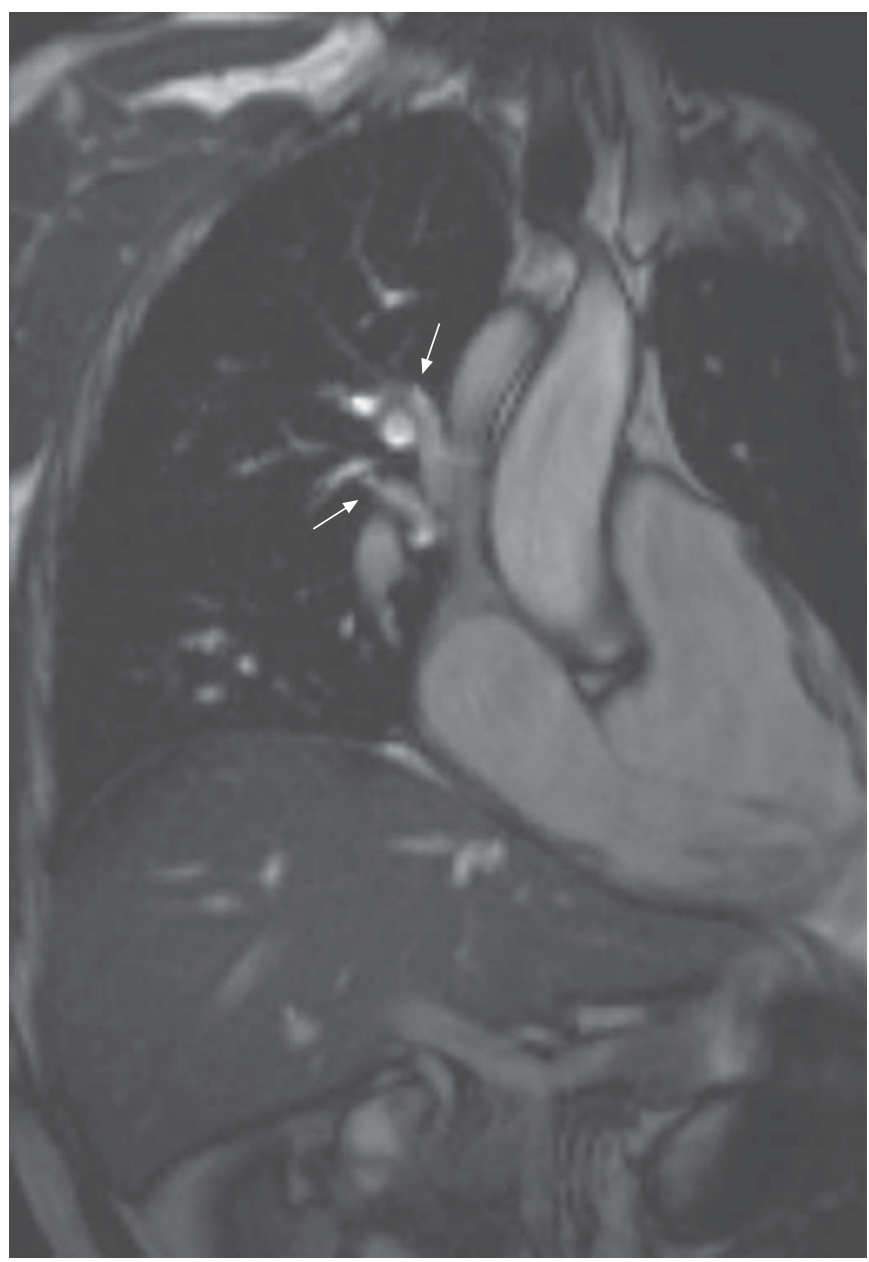

FIGURE 1. Cardiac magnetic resonance imaging demonstrates the anomalous right upper and middle pulmonary vein draining to the superior vena cava (white arrows).

increased appreciably (increase in Borg score from 0.5 to 4). Additional measures of cardiopulmonary functioning during exercise showed a peak oxygen uptake of $28.9 \mathrm{~mL} \cdot \mathrm{kg}^{-1} \cdot \mathrm{min}^{-1}$, $\sim 74 \%$ of the predicted value $\left(39.2 \mathrm{~mL} \cdot \mathrm{kg}^{-1} \cdot \mathrm{min}^{-1}\right)$, and $V \mathrm{E} /$ $V \mathrm{CO}_{2}$, which is the slope of the linear regression of the ventilation to carbon dioxide production during exercise, of 35 , suggesting reduced perfusion efficiency in this case. Heart rate increased markedly, from 73 at rest to 167 beats $\cdot \mathrm{min}^{-1}$ during exercise, and was accompanied by severe fatigue. Consistent with the 6MWT, there was no evidence of significant desaturation, with oxygen saturation decreasing only slightly, from $98 \%$ to $95 \%$ on exercise.

Based on these assessments, the patient was considered sufficiently fit and well to undergo surgery. The sinus venosus ASD was closed and the PAPVD redirected to the left atrium with an SVC baffle, while the patent foramen ovale was left open as an escape route. Intra-operative transoesophageal echocardiography confirmed the good surgical result (fig. 4). The patient made a good recovery and was discharged from hospital 6 days post-operatively. Treatment was continued with oral bosentan $125 \mathrm{mg}$ b.i.d. and aspirin $75 \mathrm{mg}$ o.d. 


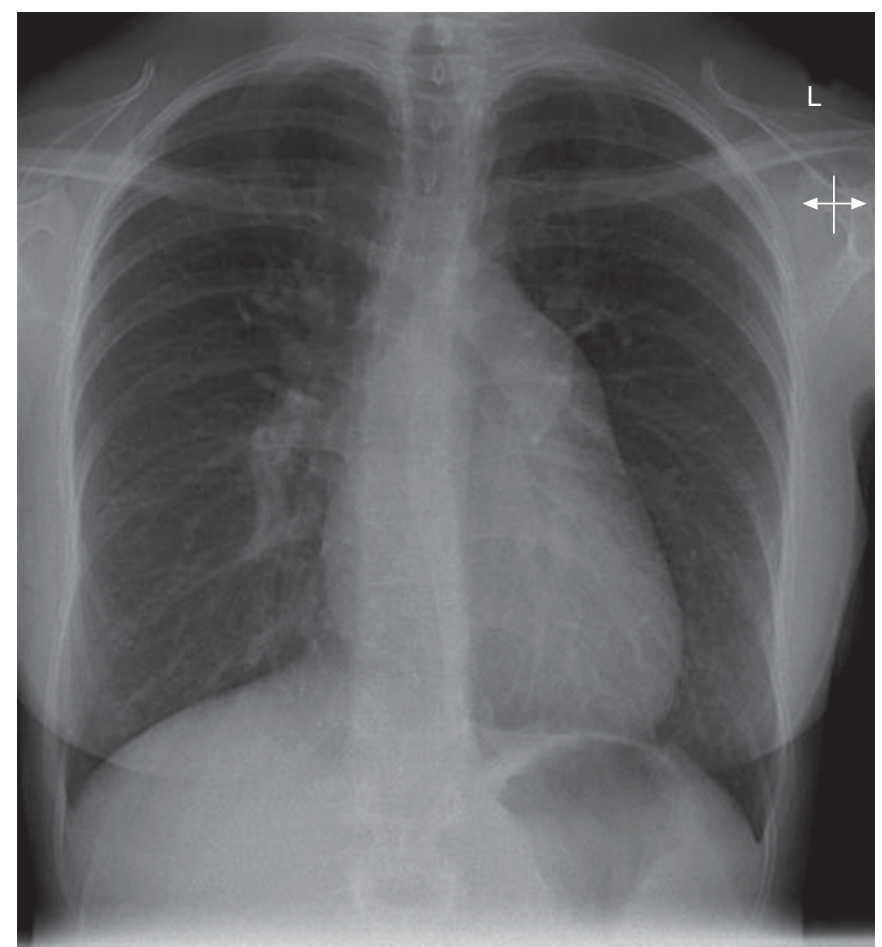

FIGURE 2. Chest radiograph.

Keen to start a family, the patient returned to the Pregnancy and Heart Disease Advisory Clinic 1 yr later. By this time the patient, who was still taking bosentan $125 \mathrm{mg}$ b.i.d. and aspirin, felt slightly better and was in WHO functional class I. Exercise capacity had increased to $500 \mathrm{~m}$ on the 6MWT and dyspnoea was appreciably better (Borg score of 0-2). Repeat RHC showed a marked improvement in haemodynamic parameters $1 \mathrm{yr}$ after surgery, with mean $P$ pa reduced to $25 \mathrm{mmHg}$ compared with $47 \mathrm{mmHg}$ pre-operatively. Under these conditions, the relative risk of major complications, including death during pregnancy or post-partum, was reduced to $5 \%$. The patient was advised that, although the risk was considerably reduced, it was not abolished, and that to further minimise risks during any pregnancy, specialist cardiac and high-risk obstetric/anaesthetic care would be needed, together with a suspension of bosentan therapy (which like other drugs in the endothelin receptor antagonist class is contraindicated during pregnancy) and step-up thromboprophylaxis.

\section{DISCUSSION}

This case demonstrates the importance of thorough clinical investigation in determining the underlying cause of $\mathrm{PAH}$, including the need for cardiac MRI, and secondly that PAH is a common mode of presentation for a sinus venosus ASD. Before referral to our centre, the patient had been diagnosed with IPAH based on her age, a significantly raised mean $P$ pa and atypical chest pain, which was probably unrelated. A positive response to adenosine challenge was the likely reason for treatment with diltiazem, although it is not unusual for calcium antagonists to fail in this setting; indeed, this would explain the switch to bosentan, a treatment that proved more effective. RHC performed at this time revealed high oxygen saturation in the pulmonary artery, an unexpected finding in this setting. Although there were signs that this patient had a cardiac shunt, because the RHC was performed and the diagnosis made without access to CHD expertise, the correct diagnosis of an ASD was missed.

Only when the patient underwent cardiac MRI after referral was the septal defect identified and the patient correctly diagnosed with a superior sinus venosus ASD with PAPVD. Interestingly, this is the most common misdiagnosis for $\mathrm{PAH}$, because sinus venosus $A S D$ is difficult to diagnose without expertise in congenital heart disease (CHD). Transthoracic echocardiography confirmed the diagnosis, although there was a difference in the size of the septal defect in comparison with the MRI scan (20 mm versus $12 \mathrm{~mm}$, respectively). This suggests that in addition to MRI, good quality transthoracic echocardiography should be mandatory in cases of suspected PAH.

With a confirmed diagnosis, a number of treatment options could be considered for this patient, who displayed a significant left-to-right shunt at rest that augmented with vasodilation.
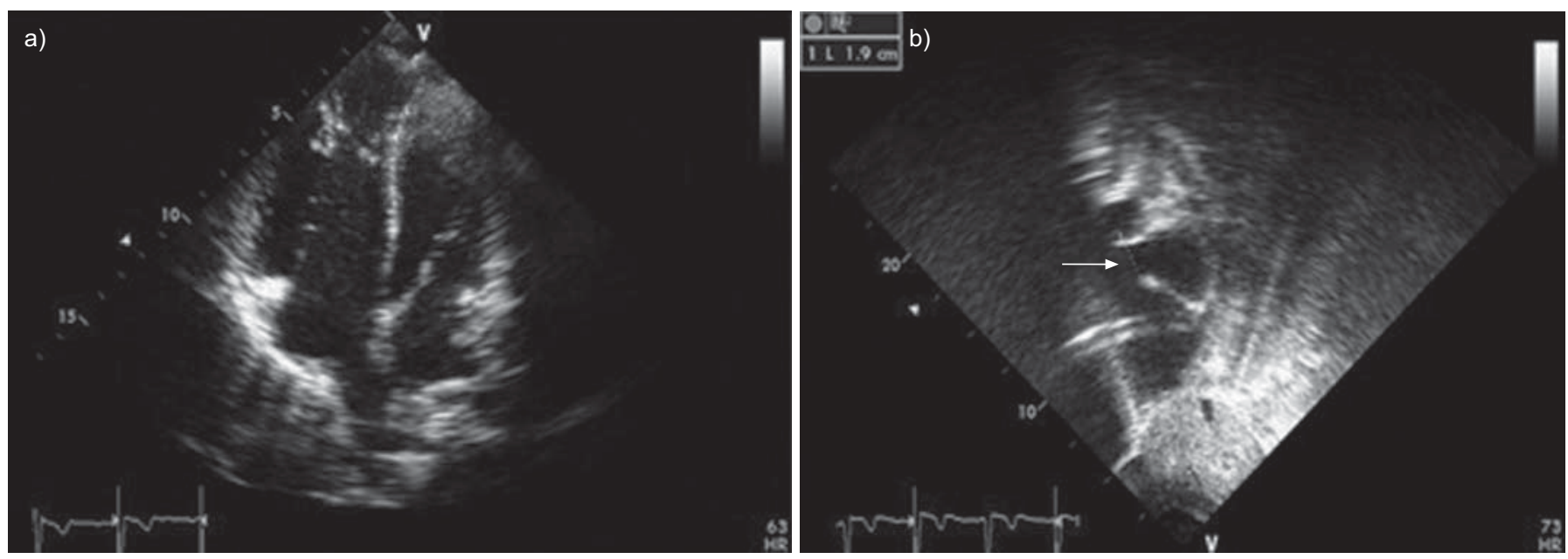

FIGURE 3. Transthoracic echocardiography showing a) right heart dilatation in the apical four-chamber view and b) the sinus venosus atrial septal defect in the subxiphoid paediatric view (white arrow) measuring $1.9 \mathrm{~cm}$ at its maximal diameter. 
TABLE 2 Pre-operative haemodynamic parameters following right heart catheterisation

\begin{tabular}{|c|c|c|c|c|}
\hline & \multicolumn{2}{|c|}{ At rest } & \multicolumn{2}{|c|}{ Vasoreactivity challenge $^{\#}$} \\
\hline & Saturation \% & Mean pressure $\mathrm{mmHg}$ & Saturation \% & Mean pressure $\mathrm{mmHg}$ \\
\hline Inferior vena cava & 74 & & 84 & \\
\hline High superior vena cava & 87 & & 68 & \\
\hline Mid right atrium & 79 & 13 & & \\
\hline Right ventricle & 83 & 16 & & \\
\hline Pulmonary artery & 82 & 47 & 96 & 44 \\
\hline Left ventricle & 96 & 12 & 99 & 11 \\
\hline Cardiac output & \multicolumn{2}{|c|}{$5.0 \mathrm{~L} \cdot \mathrm{min}^{-1}$} & & \\
\hline
\end{tabular}

Options included maintenance of existing advanced therapy, surgical repair of the defect, and surgical repair with continued advanced therapy or with advanced combination therapy. The decision to proceed with surgery was reached on the basis of a number of criteria, despite the unusually high mean $P$ pa relative to the patient's age and type of defect. A previous study in 213 patients with ASD, which included cases of sinus venosus ASD, assessed baseline mean $P$ pa levels with respect to age. In this population of patients with large left-to-right shunts but compliant pulmonary vascular beds and no advanced $\mathrm{PAH}$, there was a significant correlation between age and baseline mean $P$ pa, in which the $P$ pa value was approximately one-half the numerical value of chronological age in years [1]. Thus, for our patient aged 31 yrs, the expected mean $P$ pa was $15 \mathrm{mmHg}$ and not $47 \mathrm{mmHg}$, as observed.

In addition, a number of other factors favoured surgical repair. There was no evidence of desaturation, including during exercise, or of an abnormally high haemoglobin level that would suggest secondary erythrocytosis, or of cyanosis. The latter two are typical symptoms of Eisenmenger's syndrome (i.e. shunt reversal), a contraindication for surgery. The patient exhibited vasoreactivity and decreasing $P$ pa and PVR on challenge, both of which are good signs for operability. The resting ECG showed a normal sinus rhythm, contrary to what is often seen in sinus venosus defects where the defect itself occupies the area of the sinus node. Size of defect and degree of right heart dilatation are important determinants of operability, neither of which constituted a significant problem in this case. A pulmonary to systemic blood flow ratio of 1.9 at rest was also a good sign for surgery.

In this case, the decision to proceed with surgical repair and maintenance therapy with bosentan and aspirin proved successful, and the patient experienced a concomitant improvement in exercise capacity, dyspnoea and mean $P$ pa. In fact, 1 yr after surgery, mean $P$ pa was almost one-half the pre-operative level.

Although, over the past $10 \mathrm{yrs}$, there has been a decrease in the risk of serious complications and death associated with pregnancy in patients with $\mathrm{PAH}$, the risk remains and is especially high in the few days post-partum [2]. Before a patient such as the one described here can be advised to proceed with a pregnancy, it is essential to repeat haemodynamic assessments
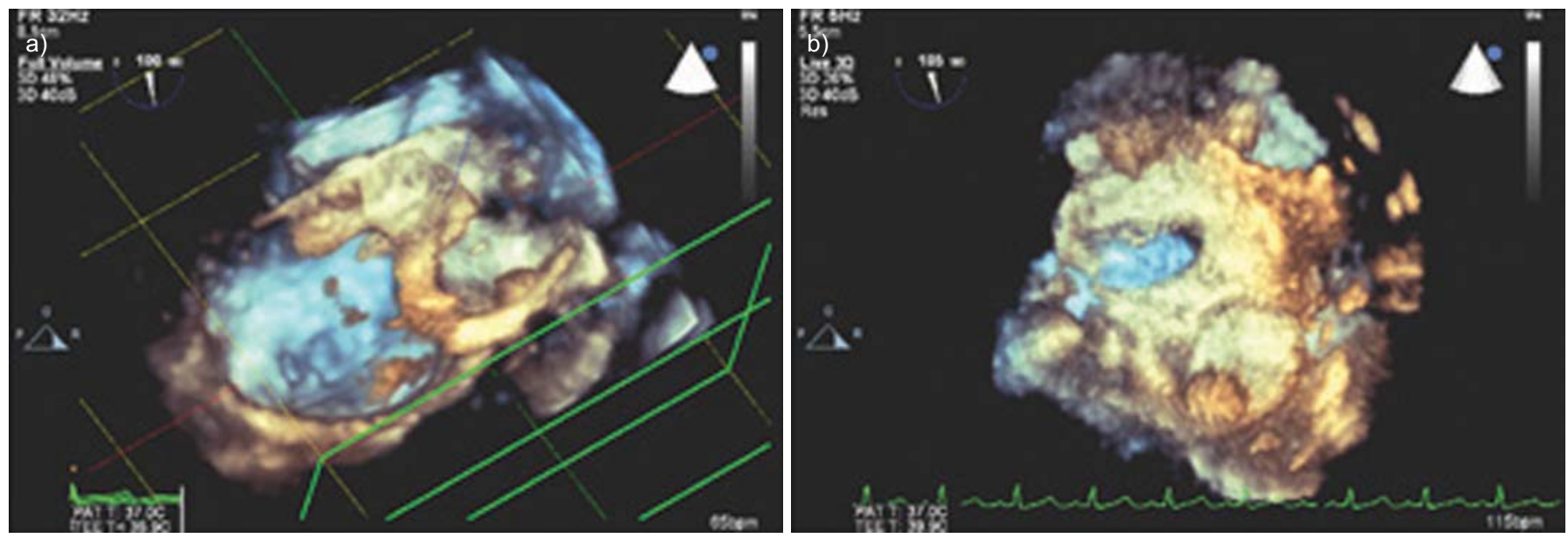

FIGURE 4. Three-dimensional trans-oesophageal echocardiography showing the sinus venosus atrial septal defect a) before and b) after surgical closure. 
and then gauge overall risk. The long-term outcome in this case, in which the patient underwent a "fenestrated" closure, is unclear, but it is encouraging that mean Ppa fell significantly while on continued bosentan therapy. Patients such as this should probably remain under tertiary care and advanced therapy for life.

In conclusion, this case study highlights the importance of secondary PAH in sinus venosus ASD, and the need to exclude a sinus venosus ASD where there is unexplained right ventricular dilatation. Various criteria can be used to determine whether a patient with a diagnosis of sinus venosus ASD is suitable for surgery; a disproportionately high mean $P$ pa relative to the patient's age and defect was an important consideration in this case. In general, these defects should be closed when there is no evidence of a right-to-left shunt and/ or presence of cyanosis at rest and more so during exercise. Ideally, mean $P$ pa with compliant pulmonary vascular beds should be about one-half the numerical value of the patient's age in years.

\section{STATEMENT OF INTEREST}

M.A. Gatzoulis has served the advisory board of Actelion UK, Pfizer and GSK, and has received unrestricted educational funds from Actelion UK and Pfizer. G. Giannakoulas has been involved in a scientific advisory board for Actelion and in addition has lectured for Actelion.

\section{ACKNOWLEDGEMENTS}

We received editorial assistance from L. Thomas, Elements Communications Ltd (Westerham, UK), supported by Actelion Pharmaceuticals Ltd (Allschwil, Switzerland).

\section{REFERENCES}

1 Gatzoulis MA, Freeman SC, Webb GD, et al. Atrial arrhythmia after surgical closure of atrial septal defects in adults. N Engl J Med 1999; 340: 839-846.

2 Bédard E, Dimopoulos K, Gatzoulis MA. Has there been any progress made on pregnancy outcomes among women with pulmonary arterial hypertension? Eur Heart J 2009; 30: 256-265. 